\title{
DARK SPOT DETECTION USING INTENSITY AND THE DEGREE OF POLARIZATION IN FULLY POLARIMETRIC SAR IMAGES FOR OIL POLUTION MONITORING
}

\author{
F. Zakeri, J. Amini ${ }^{\mathrm{a}}$
}

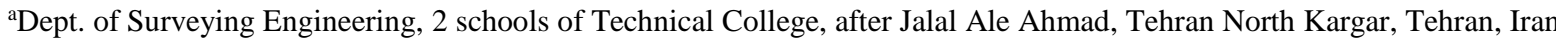
- (fatemeh.zakeri,jamini)@ut.ac.ir
\end{abstract}

KEY WORDS: Polarimetry, Synthetic Aperture Radar (SAR), Oil pollution, Degree of polarization, Dark-spot detection

\begin{abstract}
:
Oil spill surveillance is of great environmental and economical interest, directly contributing to improve environmental protection. Monitoring of oil spills using synthetic aperture radar (SAR) has received a considerable attention over the past few years, notably because of SAR data abilities like all-weather and day-and-night capturing. The degree of polarization (DoP) is a less computationally complex quantity characterizing a partially polarized electromagnetic field. The key to the proposed approach is making use of DoP as polarimetric information besides intensity ones to improve dark patches detection as the first step of oil spill monitoring. In the proposed approach first simple intensity threshold segmentation like Otsu method is applied to the image. Pixels with intensities below the threshold are regarded as potential dark spot pixels while the others are potential background pixels. Second, the DoP of potential dark spot pixels is estimated. Pixels with DoP below a certain threshold are the real dark-spot pixels. Choosing the threshold is a critical and challenging step. In order to solve choosing the appropriate threshold, we introduce a novel but simple method based on DoP of potential dark spot pixels. Finally, an area threshold is used to eliminate any remaining false targets. The proposed approach is tested on L band NASA/JPL UAVSAR data, covering the Deepwater Horizon oil spill in the Gulf of Mexico. Comparing the obtained results from the new method with conventional approaches like Otsu, K-means and GrowCut shows better achievement of the proposed algorithm. For instance, mean square error (MSE) 65\%, Overall Accuracy 20\% and correlation 40\% are improved.
\end{abstract}

\section{INTRODUCTION}

Marine oil pollution is of great environmental and economical interest, since it affects life cycle and the food chain. Detection and early warning to avoid risks caused by oil pollution due to their adverse effects is essential. Remote Sensing can improve oil spill monitoring also is a great help in early oil pollution detection. Microwave remote sensing especially Synthetic Aperture Radar (SAR) is of great interest in oil pollution monitoring due to its day and night and all-weather capturing (Brekke and Solberg 2005).

Due to the fact that oil spills reduce backscattering from the sea surface, oil slicks appear as dark regions on SAR imagery which contrast with the surrounding water surface (Topouzelis 2008). However, other phenomena which are known as look-alikes such as low-wind areas, rain cells and so on can generate dark patches on the sea clutter (Alpers et al. 1991). In addition, other factors like wind speed and incident angle and so on can affect the visibility of oil spills (Solberg and Volden 1997).

Automatic detection of oil skills can be divided into three steps: (1) dark spot detection, (2) feature extraction, and (3) classification (Brekke and Solberg 2005). In manually approach a well expert operator must to search the entire image to first find dark patches and then recognize whether they are oil spills or look-alikes. Manually approach is timeconsuming. In addition, the accuracy and precision of detection depend on the skills of the operator (Shu et al. 2010). Recently, many researchers have been interested in improving and developing new automated monitoring oil spill methods (Solberg et al. 1999), (Nirchio et al. 2005), (Solberg et al. 2003) and (Marghany 2014). 
In this research our interest is on dark spot detection. This step is the prerequisite for other steps. In addition, it is critical and time-consuming one which affects other step accuracy. Furthermore, as long as dark patches cannot be detected properly, oil spills can never be detected at other steps.

Recently many attempts have been made to automate the process of detecting dark patches. Theresholding algorithm is a conventional one in this step. Theresholding method can be divided into two general ones: 1) global thresholding and 2) adaptive thresholding. In global approach, a universal threshold is used for the entire image (Nirchio et al. 2005) and (Chang et al. 2008). In adaptive method, the threshold is selected locally (Solberg et al. 2007). In addition, recently other methods same as neural network (Topouzelis et al. 2008), wavelet based methods (Kuzmanić and Vujović 2010), classification based methods like support vector machines (Mercier and Girard-Ardhuin 2005) and so on have been introduced.

Introduced algorithms detect dark patches based on intensity property. There is, nevertheless, two main difficulties that occur when using intensity feature: (1) SAR images contain speckles resulting from the coherent nature of incident energy. Due to the speckle effects, intensity values may show considerable variability, even in a uniform region (Oliver and Quegan 2004). (2) Based on the local sea state, the type of the oil spill, the resolution and incident angle of the SAR imagery, the contrast between the oil spills and sea clutter can vary (Topouzelis 2008). These difficulties can interrupt getting to the comprehensive and fast solution.

RADAR polarimetry particularly full polarimetric images can provide complementary information besides intensity ones. Polarimetric data provide useful information to detect oil spills. Different polarizations produce different interactions between the signal and the scene and only fully polarimetric measures allow collecting all the information (Guissard 1994), (Lee et al. 2001). When wind conditions are low to moderate and imaging is performed at the intermediate incident angles, Bragg scattering mechanism occurs in slick-free and the weak-damping slick-covered sea (e.g. biogenic look-alikes) while, in case of oil-covered sea surface, a completely different, i.e. non-Bragg, scattering mechanism is in place. By using some polarimetric features same as the amount of unpolarized backscatted energy, the correlation between co-polarized channels and the polarimetric randomness, the departure from Bragg scattering can be measured (Migliaccio et al. 2007), (Migliaccio et al. 2009b), (Migliaccio et al. 2009a), (Migliaccio et al. 2011), (Velotto et al. 2011) and (Nunziata et al. 2013).

In this paper a new method is proposed by combining intensity and polarization information to can improve dark spot detection which is the first step of the oil spill monitoring. The proposed approach is tested on $\mathrm{L}$ band NASA/JPL UAVSAR data, covering the Deepwater Horizon oil spill in the Gulf of Mexico.

The remaining of this paper is organized as follows. In section 2 , the proposed method is described while, in section
3 , the results of some experiments are presented and discussed. The conclusion is drawn in section 4.

\section{MATERIALS AND METHODS}

\subsection{Principles involved}

The proposed method is based on the intensity and polarization information. As was mentioned due to reduction of the arrived signal from oil spills, these patches appear darker than surrounding areas. So, by using a simple thresholding darker areas can be separated. But as was mentioned in the introduction section, intensity and contrast between the oil spills and surrounding vary due to the speckles, sea state, resolution and incident angle. Due to the mentioned reasons using the polarimetric information can produce other information source to can detect dark areas more effectively.

Degree of polarization (DoP) is an important feature characterizing a partially polarized electromagnetic wave (Wolf 1959). DoP has some definitions in terms of the coherence matrix elements, eigenvalues of the coherence matrix and Stokes parameters (Massonnet and Souyris 2008).

DoP is defined as the ratio of the power density of the polarized part of the wave to the total power density carried by the wave. DoP is between 0 and 1 (Massonnet and Souyris 2008):

$$
D o P=\left(1-4 \frac{|J|}{(t r J)^{2}}\right)^{\frac{1}{2}}
$$

where $J$ is known as the wave coherence matrix

The Barakat DoP is one of the generalization of the DoP. For a full polarimetric SAR, and under the scattering reciprocity, the Barakat DoP (B_DoP) is expressed as (Shirvany 2012):

$$
B_{-} D o P=\left(1-27 \frac{|J|}{(t r J)^{3}}\right)^{\frac{1}{2}}
$$

As was mentioned in the introduction section, Bragg scattering occurs in slick free sea state which has low depolarization. So, DoP must close to 1 .While, non-Bragg scattering occurs in case of oil-covered sea surface. So, DoP must close to 0 (Migliaccio et al. 2009b).

\subsection{Proposed Algorithm}

Figure 1 shows the embedding procedure of this proposed method. The proposed method has two main steps. At the first step the focus is on the intensity information and an intensity threshold segmentation is implemented. Potential dark patch pixels which were passed from the first test will enter to the second step. At this step, the DoP of the pixels is estimated. A DoP threshold is selected and pixels with DoP below the threshold are regard as the real dark patches. The entire steps and details are described as follows. 


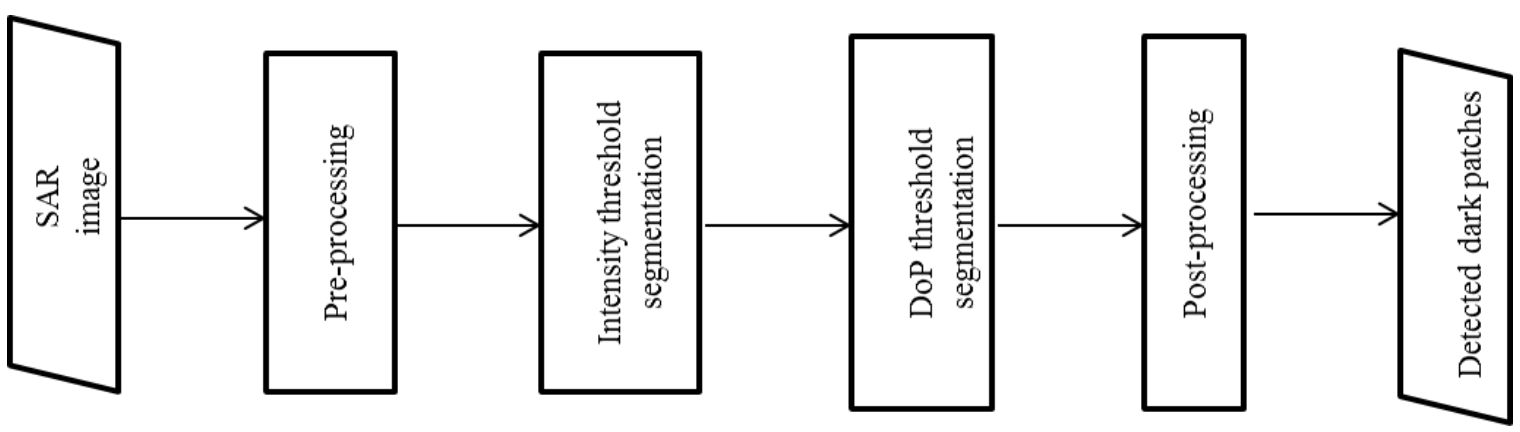

Figure 1. flowchart of the proposed method

First, a $3 \times 3$ Gaussian filter with a standard deviation of 0.5 is used to reduce the speckle effects of the span image. This results in a more stable intensity threshold (Shu et al. 2010). Then, the histogram of the intensity image is adjusted using a piecewise linear transform. The lowest $1 \%$ of all pixel values are specified as 0 , while the highest $1 \%$ are specified as 255 . The remaining pixel values are stretched within the range from 0 to 255 using a linear transformation. This causes enhancing contrast between dark patches and the background.

Second, In order to find an intensity threshold that maximizes the ratio of the between-calss variance to the within-class variance, the Otsu method is used due to the simplicity and effectiveness (Otsu 1975). Pixels below this threshold are considered as dark areas.

Third, the DoP of the potential dark areas is computed by the $7 \times 7$ averaging window. In the previous studies the DoP threshold was set to 0.45 (Nunziata et al. 2013). While this threshold can verify with different oil types or sea states, a new DoP threshold estimation is introduced. In order to determine the threshold value, the average of the DoP of potential dark patches is computed. This threshold value is applied to the DoP image and potential dark pixels which their DoP degree is under the DoP threshold are considered as the dark pixels.

Finally, due to the incidental errors, some regions may have been incorrectly detected as dark spots. An area threshold is used to reduce these false targets. Regions with area under 100 pixels are regarded as the false alarms.

\section{EXPRIMENTAL RESULTS}

In this section, the results obtained by processing the SAR images using the proposed method are presented. The proposed approach is tested on the $\mathrm{L}$ band NASA/JPL UAVSAR data, covering the Deepwater Horizon oil spill in the Gulf of Mexico to test the reliability and efficiency of the algorithm. The dataset was downloaded from NASA's Alaska SAR Facility (ASF). The algorithms were implemented in MATLAB 2012.

The experimental result is also compared with Otsu, Kmeans and GrowCut. To investigate the efficiency of the proposed method, three criteria are used: Mean Square Error (MSE), Overall Accuracy and the correlation between the output image and the image of manual segmentation.
Figure 2(a)-(f) show the original image and segmented images by the proposed method and other mentioned methods. Extracted dark regions are displayed in black. As can be seen in Figure 2, the proposed method is able to detect regions which have small amounts contrast with background. In addition, the number of false alarms is very high in the background in other methods.

To test the capability of the proposed method for detecting dark region boundaries, different obtained boundary images by Canny operator is compared in Figure 3(a)-(e). Visual inspection shows the extruded boundary from proposed algorithm and the real dark spot boundary match quite well.

The mentioned criteria were calculated on a subset of the dataset. Table 1 summarizes the assessment parameters. In Table 1, the best value in each column is set in boldface. By comparing the values of Table 1 one can conclude that due to the using polarimetry information besides intensity ones the proposed method achieves better results.

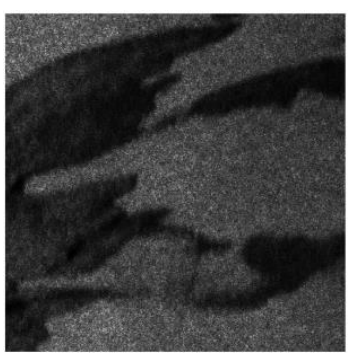

(a)

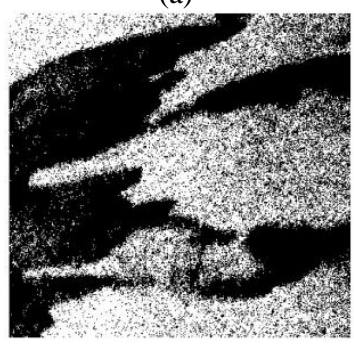

(c)

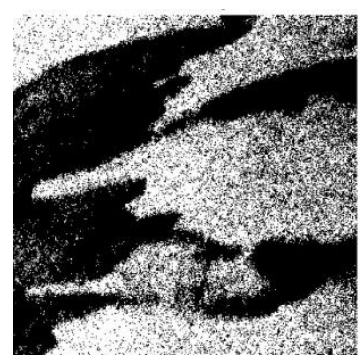

(b)

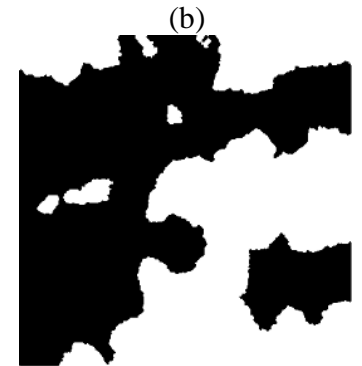

(d) 


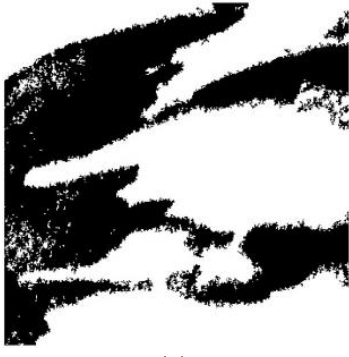

(e)

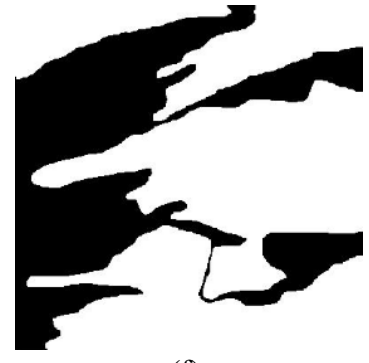

(f)
Figure 2. Segmentation of different methods: (a) Original image, (b)Otsu, (c) K-means, (d) GrowCut, (e) Proposed, (f) Ground truth

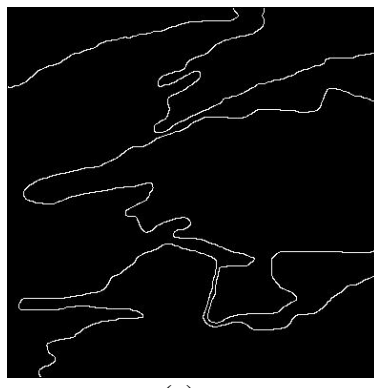

(a)

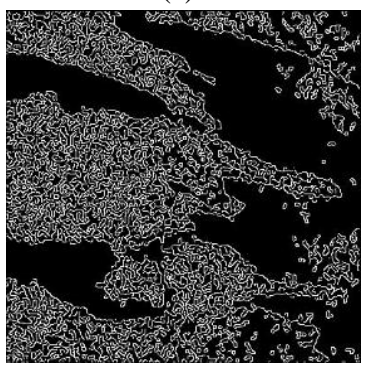

(c)

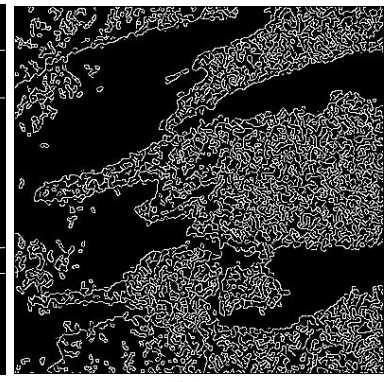

(b)

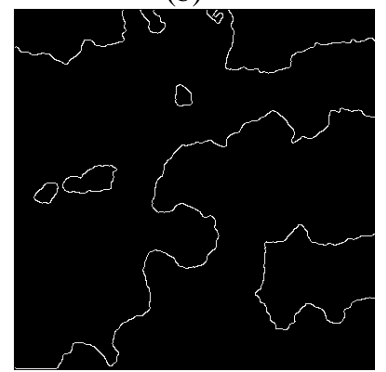

(d)

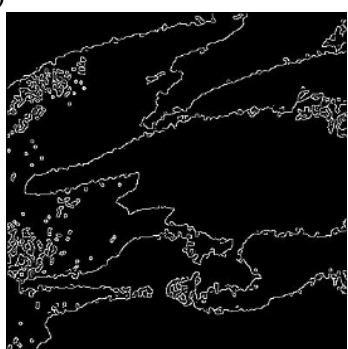

(e)

Figure 3. Extracted edges by Canny operator: (a) Ground truth, (b) Otsu, (c) K-means, (d) GrowCut, (e) Proposed

Table 1 Segmentation results of UAVSAR oil spill image

\begin{tabular}{|c|c|c|c|}
\hline & MSE & OA & CORR \\
\hline DoP_B & $\mathbf{0 . 0 8 3 9}$ & $\mathbf{0 . 9 1 6 1}$ & $\mathbf{0 . 8 2 8 9}$ \\
\hline Span_K_means & 0.2394 & 0.7606 & 0.5931 \\
\hline GrowCut & 0.2127 & 0.7873 & 0.6211 \\
\hline Span_Otsu & 0.2413 & 0.7587 & 0.5909 \\
\hline
\end{tabular}

\section{CONCLUSION}

The first step in oil spill monitoring is the detection of dark region which its precision affect other steps' precision. In this paper, a new algorithm based on the polarimetry information as well as intensity ones to detect dark patches in SAR images is presented. The power of the degree of the polarization and the intensity information with together is demonstrated to detect dark patches. The proposed method in comparison with other mainly used segmentation methods like Otsu, K-means and GrowCut is tested on L band NASA/JPL UAVSAR data, covering the Deepwater Horizon oil spill in the Gulf of Mexico. The results show better achievement of the proposed method. For instance, mean square error (MSE) 65\%, Overall Accuracy 20\% and correlation $40 \%$ are improved. Degree of polarization has some other definitions. This motivates us to compare these definitions in future work.

\section{REFRENCES}

Alpers, W., Wismann, V., Theis, R., Hühnerfuss, H., Bartsch, N., Moreira, J., \& Lyden, J.D. (1991). The damping of ocean surface waves by monomolecular sea slicks measured by airborne multi-frequency radars during the SAXON-FPN experiment. In, Geoscience and Remote Sensing Symposium, 1991. IGARSS'91. Remote Sensing: Global Monitoring for Earth Management., International (pp. 1987-1990): IEEE

Brekke, C., \& Solberg, A.H. (2005). Oil spill detection by satellite remote sensing. Remote Sensing of Environment, 95, 1-13

Chang, L., Tang, Z., Chang, S., \& Chang, Y.-L. (2008). A region-based GLRT detection of oil spills in SAR images. Pattern Recognition Letters, 29, 1915-1923

Guissard, A. (1994). Mueller and Kennaugh matrices in radar polarimetry. Geoscience and Remote Sensing, IEEE Transactions on, 32, 590-597

Kuzmanić, I., \& Vujović, I. (2010). Oil spill detection in SAR images using wavelets and morphology. In, ELMAR, 2010 PROCEEDINGS (pp. 337-340): IEEE

Lee, J.-S., Grunes, M.R., \& Pottier, E. (2001). Quantitative comparison of classification capability: fully polarimetric versus dual and single-polarization SAR. Geoscience and Remote Sensing, IEEE Transactions on, 39, 2343-2351

Marghany, M. (2014). Utilization of a genetic algorithm for the automatic detection of oil spill from RADARSAT-2 SAR satellite data. Marine pollution bulletin, 89, 20-29

Massonnet, D., \& Souyris, J.-C. (2008). Imaging with synthetic aperture radar. CRC Press

Mercier, G., \& Girard-Ardhuin, F. (2005). Oil slick detection by SAR imagery using support vector machines. In, Oceans 2005-Europe (pp. 90-95): IEEE

Migliaccio, M., Gambardella, A., Nunziata, F., Shimada, M., \& Isoguchi, O. (2009a). The PALSAR polarimetric mode for sea oil slick observation. Geoscience and Remote Sensing, IEEE Transactions on, 47, 4032-4041

Migliaccio, M., Gambardella, A., \& Tranfaglia, M. (2007). SAR polarimetry to observe oil spills. Geoscience and Remote Sensing, IEEE Transactions on, 45, 506-511

Migliaccio, M., Nunziata, F., \& Gambardella, A. (2009b). On the co-polarized phase difference for oil 
The International Archives of the Photogrammetry, Remote Sensing and Spatial Information Sciences, Volume XL-1/W5, 2015 International Conference on Sensors \& Models in Remote Sensing \& Photogrammetry, 23-25 Nov 2015, Kish Island, Iran

spill observation. International Journal of Remote Sensing, 30, 1587-1602

Migliaccio, M., Nunziata, F., Montuori, A., Li, X., \& Pichel, W.G. (2011). A multifrequency polarimetric SAR processing chain to observe oil fields in the Gulf of Mexico. Geoscience and Remote Sensing, IEEE Transactions on, 49, 4729-4737

Nirchio, F., Sorgente, M., Giancaspro, A., Biamino, W., Parisato, E., Ravera, R., \& Trivero, P. (2005). Automatic detection of oil spills from SAR images. International Journal of Remote Sensing, 26, 1157-1174

Nunziata, F., Gambardella, A., \& Migliaccio, M. (2013) On the degree of polarization for SAR sea oil slick observation. ISPRS Journal of Photogrammetry and Remote Sensing, 78, 41-49

Oliver, C., \& Quegan, S. (2004). Understanding synthetic aperture radar images. SciTech Publishing Otsu, N. (1975). A threshold selection method from gray-level histograms. Automatica, 11, 23-27

Shirvany, R. (2012). Estimation of the Degree of Polarization in Polarimetric SAR Imagery: Principles and Applications

Shu, Y., Li, J., Yousif, H., \& Gomes, G. (2010). Darkspot detection from SAR intensity imagery with spatial density thresholding for oil-spill monitoring. Remote Sensing of Environment, 114, 2026-2035

Solberg, A.H., Brekke, C., \& Husøy, P.O. (2007). Oil spill detection in Radarsat and Envisat SAR images. Geoscience and Remote Sensing, IEEE Transactions on, 45, 746-755

Solberg, A.H., Dokken, S.T., \& Solberg, R. (2003). Automatic detection of oil spills in Envisat, Radarsat and ERS SAR images. In, Geoscience and Remote Sensing Symposium, 2003. IGARSS'03. Proceedings. 2003 IEEE International (pp. 2747-2749): IEEE

Solberg, A.H.S., Storvik, G., Solberg, R., \& Volden, E. (1999). Automatic detection of oil spills in ERS SAR images. Geoscience and Remote Sensing, IEEE Transactions on, 37, 1916-1924

Solberg, A.H.S., \& Volden, E. (1997). Incorporation of prior knowledge in automatic classification of oil spills in ERS SAR images. In, Geoscience and Remote Sensing, 1997. IGARSS'97. Remote Sensing-A Scientific Vision for Sustainable Development., 1997 IEEE International (pp. 157-159): IEEE

Topouzelis, K., Karathanassi, V., Pavlakis, P., \& Rokos, D. (2008). Dark formation detection using neural networks. International Journal of Remote Sensing, 29, 4705-4720

Topouzelis, K.N. (2008). Oil spill detection by SAR images: dark formation detection, feature extraction and classification algorithms. Sensors, 8, 6642-6659

Velotto, D., Migliaccio, M., Nunziata, F., \& Lehner, S. (2011). Dual-polarized TerraSAR-X data for oil-spill observation. Geoscience and Remote Sensing, IEEE Transactions on, 49, 4751-4762

Wolf, E. (1959). Coherence properties of partially polarized electromagnetic radiation. Il Nuovo Cimento, $13,1165-1181$ 